\title{
CONFERENCE NOTICE
}

\section{Call for Papers: Vision 2006}

The Papers Committee of the South African Optometric Association invites you to participate in the Vision 2006 programme by submitting your papers and/or workshops presentation for consideration. No specific theme is relevant for Vision 2006, however, papers, posters and workshops are invited in any of the following areas: Binocular Vision, Cornea and Contact Lenses, Child Vision and Development, Dispensing, Low Vision, Ocular Disease, Ophthalmic Optics, Optics, Optometric Education, Public Health, Practice Management, Sports Vision, and Visual Science.

Submissions for papers, case reports and workshops will only be accepted via the website http://www.saoa.co.za. The decision of the Papers Committee will be final and no correspondence will be entered into after finalization of the program. The deadline for submission is 30 November 2005.

\section{Papers}

1. Authors must specify the type of paper being presented:

- Case Report Paper

- Educational Paper

- Literature review

- Scientific Paper, including research reports

- Case Report - digital photography NEW!

2. Submissions of abstracts need to include the following:

- Name, Qualifications, Short CV and Contact Details of presenter

3. For scientific papers and research reports, the following information needs to be included:

- Title and Author(s)

- Presentation preference: Oral presentation or workshop
- Abstract text including Purpose, Methods, Results and Conclusion

- Keywords

4. For Educational and Case Report Papers or workshops, the following need to be included:

- Title and Author(s)

- Presentation preference: Oral presentation or workshop

- Abstract text including Background, Case report and Conclusions

- Keywords

5. Authors need to declare affiliations if sponsored for the paper or workshop presentation. Abstracts should not be more than 500 words (and any special requirements should be clearly stated, for example, audio-visual media should be able to handle video clips, etc).

6. Indicate time allocation needed:

- 25 minutes with 5 minutes for questions

- 50 minutes with 5 minutes for questions (preferable)

Workshops (recommended time for a workshop is 2 to 3 hours): A complete submission includes the following:

1. Course outline (one page) including Topic, Description of Workshop Outcomes, Short Description of Content and Workshop Duration

2. Name, Short CV and Contact Details of presenter and/or co-presenters

3. Equipment requirements

\section{NEW: Case Reports - digital photography}

You are invited to submit digital photograph(s) taken of ocular pathology to be presented in the form of a workshop. Each presentation will be 
about 15 minutes long. The workshop will take the form of:

- Presentation of digital photography

- Discussion of diagnosis

- Discussion of treatment options

Submit the following information:

6.1. Name, Short CV and Contact Details of presenters and co-presenters

6.2. Title of ocular pathology presented

6.3. Digital photograph(s) should be submitted in jpeg format. 